\title{
Optical properties of graphene film growing on a thin copper layer
}

\author{
T.S. Rozouvan ${ }^{1}$, L.V. Poperenko ${ }^{1}$, V.G. Kravets ${ }^{2}$ and I.A. Shaykevich ${ }^{1}$ \\ ${ }^{I}$ Taras Shevchenko National University of Kyiv, Department of Physics, \\ 2, prospect Glushkova, 03187 Kyiv, Ukraine \\ ${ }^{2}$ School of Physics and Astronomy, University of Manchester, Manchester, M13 9PL, UK
}

\begin{abstract}
Optical properties and surface structure of graphene films grown on thin 1- $\mu \mathrm{m}$ copper layer using the chemical vapor deposition method were investigated applying spectroscopic ellipsometry and nanoscopic measurements. Angle-variable ellipsometry measurements were performed to analyze features of optical conductivity and dispersion of the complex refraction index. Significant enhancement of the absorption band in the graphene single layer with respect to the bulk graphite was observed due to interaction between excited localized surface plasmon at the surface of thin $\mathrm{Cu}$ layer and graphene's electrons. Scanning tunneling microscopy measurements with atomic spatial resolution revealed vertical crystal lattice structure of the deposited graphene layer. The obtained results provide direct evidence of the strong influence of the growth conditions on electronic and optical behavior of graphene films.
\end{abstract}

Keywords: graphene, optical properties, Raman spectroscopy, ellipsometry, scanning tunneling microscopy.

Manuscript received 22.12.15; revised version received 24.02.16; accepted for publication 16.03.16; published online 08.04.16.

\section{Introduction}

Graphene monolayers exhibit unique properties due to conjugation of $\pi$-electrons along all honeycomb crystal lattice and 2D structures density of states with "ultrarelativistic" electrons and holes. These unique characteristics allow graphene to be used as a basic material in a variety of different scientific projects. Some of these material properties, including high electrical and heat conductivity as well as its capability to keep stability at room temperature, make it an attractive candidate for nanoscale devices. Microchips that are made using graphene can operate in the terahertz frequency range. Another key attribute includes its material properties being dependent on the substrate ones. The substrate atoms diffuse to the graphene layer and change its local properties. A copper substrate can be used as an electrode in nanoscale devices because of the copper high electrical conductivity and relatively low price. Large single-layer graphene films ranging in size of centimeters can be grown on copper substrates by using chemical vapor deposition [1]. A number of interesting graphene applications includes its use in optoelectronic devices. A sensitive and selective biosensor is demonstrated using vertically-oriented graphene sheets that are grown on the sensor electrode [2]. Copper films covered with one to several graphene layers may exhibit distinct plasmonic characteristics. They demonstrate stable characteristics and can be also used in plasmonic devices [3]. Plasmons in a graphenemetallic substrate system may display a rather complicated picture. For example, the electromagnetic response of graphene and the spectrum of collective plasmon excitation were studied as a function of the 
wave vector and frequency [4]. The quasi-particle dynamics of the sheet plasmons in epitaxially grown on graphene layers on $\mathrm{SiC}(0001)$ was studied as a function of temperature, intrinsic defects, influence of multilayers and carrier density [5]. Graphene can be used as transient electrodes in computer screens because of its high optical transmittance and simultaneous high electrical conductivity. Using the ellipsometric measurements with consecutive numerical simulations, we can determine optical constants (incl. refraction index $n$ and absorption coefficient $k$ ) from ellipsometric data for a single graphene layer in a broad spectral range. These data can indicate the graphene optical transmittance as well as its optical conductivity at high frequencies. In this work, we performed spectral ellipsometric measurements on a graphene layer deposited on a copper substrate.

\section{Experimental}

In this work, we performed scanning tunneling microscopy experiments with graphene deposited on a copper layer to understand surface morphology of studied samples. Large area $1 \times 1 \mathrm{~cm}^{2}$ of high quality graphene film was grown on copper $1-\mu \mathrm{m}$ layer by using the chemical vapor deposition (CVD) method [6]. Just before use, all silica substrates were ultrasonically cleaned in heated acetone and then in ethanol. We also deposited thin adhesive layer of $\mathrm{Cr}$ with the thickness about $3 \mathrm{~nm}$ on the substrates before growing the $\mathrm{Cu}$ films. The substrates with the deposited $\mathrm{Cu}$ layer were inserted into a silica tube and heated up to $1000^{\circ} \mathrm{C}$. Then, a gas mixture of hydrogen $\mathrm{H}_{2}$ and methane $\mathrm{CH}_{4}$, with flow rates of 20 and 40 cubic centimetres per minute, respectively, were introduced into the CVD chamber with keeping the growth temperature near $1000{ }^{\circ} \mathrm{C}$ and reactor pressure of $600 \mathrm{mTorr}$ for $30 \mathrm{~min}$. Finally, the CVD chamber was rapidly cooled to room temperature in hydrogen atmosphere. The copper layer was deposited using e-beam evaporation system Edwards E306 supplied by Moorfield Nanotechnology Limited onto silica slides at the base pressure close to $5 \times 10^{-7}$ mbar. Growth of the metal film was monitored by a calibrated quartz microbalance, at the approximate growth rate of $0.5 \mathrm{~nm} / \mathrm{s}$.

Microscope INTEGRA NT-MDT was used for precise surface topology study, for this we used the scanning tunneling microscopy (STM) regime. Scanning tunneling microscopy spatial resolution reached up to $0.1 \mathrm{~nm}$. In order to obtain direct surface profiles, the experiments were performed in the regime of measurements with a constant tunneling current (and constant distance between the needle tip and sample surface).

In order to confirm availability of a graphene film on the top of $\mathrm{Cu}$ layer, we used Raman spectroscopy. Raman spectra were recorded with a Renishaw RM1000 spectrometer with the excitation wavelengths 514 and
$633 \mathrm{~nm}$. Spectral ellipsometric measurements were performed in $\lambda=245 \ldots 1000 \mathrm{~nm}$ spectral region by applying the Beattie technique [7]. The azimuth of restored polarization $\Psi$ and phase shift $\Delta$ between $p$ - and $s$-components were measured at various angles of incidence $\varphi$ and different values of the light wavelength $\lambda$. Ellipsometric parameters of graphene layer at different angles of incidence for the studied samples were calculated applying Airy's formula [8]:

$R_{s, p}=\frac{r_{s, p_{i}}+r_{s, p_{i+1}} e^{2 i \theta}}{1+r_{s, p_{i}} r_{s, p_{i+1}} e^{2 i \theta}}$,

where $i=1,2$ and $R_{s, p}$ is the amplitude reflection coefficient from the double copper-graphene layer (Fig. 1), and

$r_{s_{i}}=\frac{n_{i} \cos \left(\varphi_{i}\right)-n_{i+1} \cos \left(\varphi_{i+1}\right)}{n_{i} \cos \left(\varphi_{i}\right)+n_{i+1} \cos \left(\varphi_{i+1}\right)}$,

$r_{p_{i}}=\frac{n_{i+1} \cos \left(\varphi_{i}\right)-n_{i} \cos \left(\varphi_{i+1}\right)}{n_{i+1} \cos \left(\varphi_{i}\right)+n_{i} \cos \left(\varphi_{i+1}\right)}$.

Here $r_{s, p i}$ is the Fresnel amplitude reflection coefficients from the boundary separating $i$-th and $(i+1)$-th layers for $s$ - or $p$-polarization component. $\varphi_{i}$ light incidence angle from $i$-th layer to $(i+1)$-th one, which can be found from Snell's law. $\theta_{i}$ is the phase thickness of graphene layer, which can be found applying the ratio $\theta=\frac{2 \dot{\Gamma} \mathrm{O}}{{ }_{\check{r}}^{\prime} И} n_{2} d \cos \left(\varphi_{2}\right)$ where $n_{2}$ and $d$ are the $i$-th layer refraction index and its thickness, and $\lambda$ is the light wavelength. Refraction indices of the layers are complex numbers except that for air. In order to determine both refraction index $n$ and absorption coefficient $k$, we can use the relationship

$R_{p} / R_{s}=\tan \Psi \exp \Delta$.

Eqs. (1) to (3) can be solved only numerically, in our case we used MathCad software. Copper optical constants were taken from [3] and ellipsometric parameters $\Psi$ and $\Delta$ for various angles of incidence $\varphi$ were obtained experimentally.

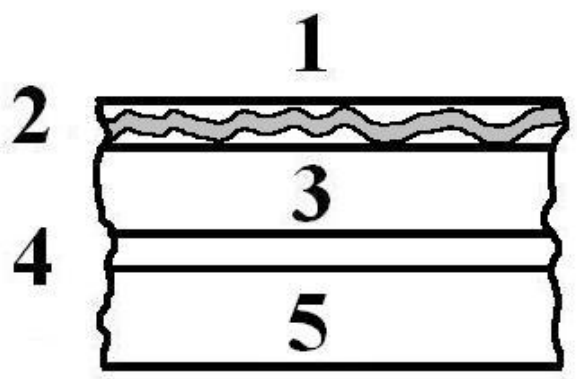

Fig. 1. Scheme of graphene layer on copper. (1) indicates air, (2) heterogeneous layer of grapheme (grey) and copper granules, (3) copper film, (4) thin adhesive Cr layer, and (5) silica substrate. 


\section{Results and discussion}

Results of STM experiments on copper with CVD graphene are presented in Fig. 2. We can see graphene crystals with straight edges, which cover the scanned area with dimensions $1 \times 1 \mu \mathrm{m}$. Scanning tunneling microscopy measurements with atomic spatial resolution revealed the vertical crystal lattice structure of the deposited graphene (Fig. 3). The spatial period of vertical muster in Fig. 3 is approximately equal to $0.63 \mathrm{~nm}$ which is close to the length of a primitive vector of graphite crystal lattice $(0.67 \mathrm{~nm})$. A vertical graphene crystal lattice schematics on a substrate plane is presented in Fig. 4. Its structure is constructed as $4 \times 1 \times 2$ graphite plane supercell without carbon atoms that have two dangling bonds. It is clear that atoms with two unsatisfied valences in the vertical graphene lattice are rather chemically active and would react with atmosphere oxygen. In Fig. 3, we observe only even chains of carbon atoms from Fig. 4, presumably those with an almost formed honeycomb structure. The odd chains are not registered in our STM experiments, because it contains no hybrid $s p^{2}$-orbitals with perpendicular to the substrate surface symmetry axes. The perpendicularly oriented hybrid orbitals provide lower electric resistance (and, as a consequence, higher electric current) during de Broglie electron wave propagation in the direction normal to the substrate surface. These orbitals can be registered in STM experiments when measuring spatial distribution of tunneling currents. The fact we registered only even vertical graphene chains proves that we observe a singlelayer vertical graphene. In the case of a few layers vertical graphene, we would register only the odd chains, because there observed is the honeycomb structure with vertically oriented $\pi$-orbitals.

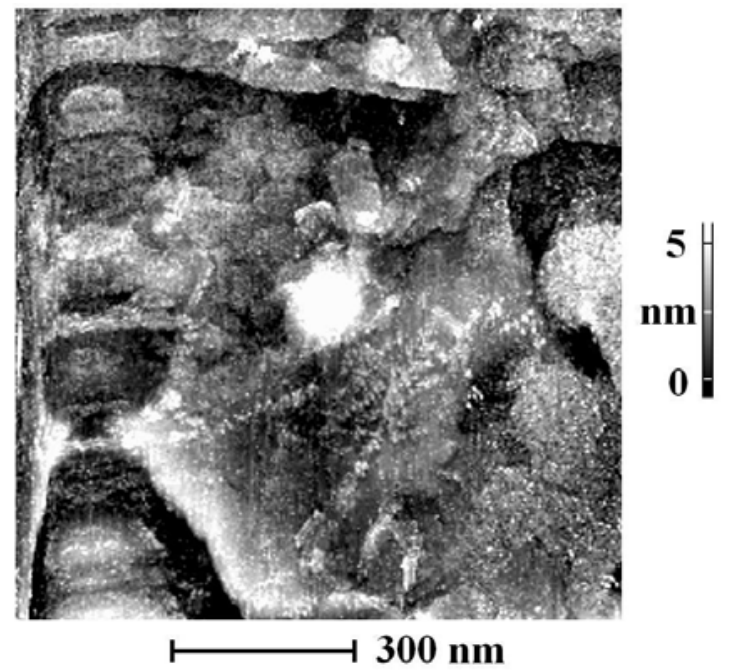

Fig. 2. STM scans of graphene on a copper substrate. Spatial resolution $4 \mathrm{~nm}$.

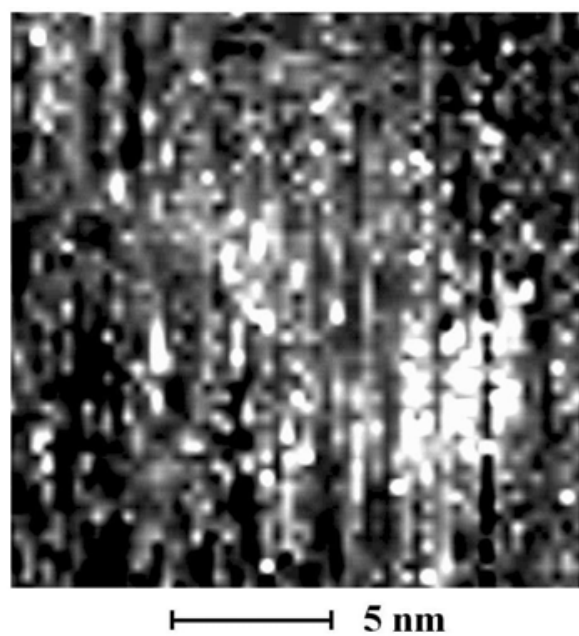

Fig. 3. STM scans of graphene on a copper substrate. Spatial resolution $0.1 \mathrm{~nm}$.

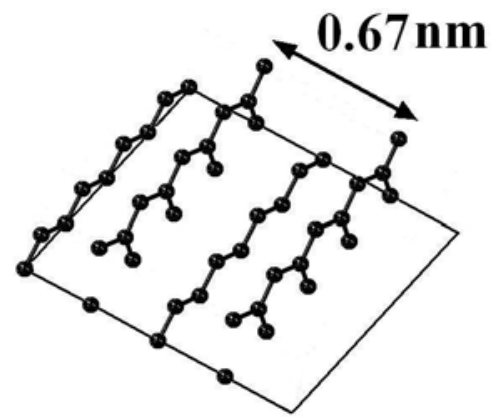

Fig. 4. Crystal lattice of single-layer vertical graphene on a substrate.

The Raman signature of graphene (see Fig. 5) consists of a set of distinct peaks: the $G$ peak at $1580 \mathrm{~cm}^{-1}$ caused by the in-plane vibrational $E_{2 G}$ mode at the Brillouin zone center and the $2 D$ peak near $2700 \mathrm{~cm}^{-1}$ caused by two-phonon inter-valley scattering [9]. We can conclude that the films are predominantly single-layer graphene (SLG) without defects (Fig. 5). These results are important, because Raman spectroscopy based on inelastic light scattering is the main instrument to identify single-layer graphene and to monitor doping, defects, disorder, edges, strain and chemical modification of SLG [9]

Results of numerical simulation for the refraction index $n$, absorption coefficient $k$ and optical conductivity for graphene thin film on copper are presented in Figs 6 to 8 . Dispersion curves for pure graphite taken from [10] are also presented in these figures. As we can see from Fig. 8, the refraction index dispersion curves for graphite and graphene layers are similar, and refraction index values for both substances are almost identical. By contrast, the absorption coefficient curves in Fig. 7 vary. Graphite has a small maximum in the vicinity of $650 \mathrm{~nm}$. The graphene dispersion curve has a distinct absorption band approximately in the same spectral region. Let us take the optical conductivity: 
$\sigma(\omega)=n k \omega / 2 \pi$,

where $\omega$ is the light frequency. Dispersion curves $\sigma(\omega)$ for the graphene thin film and for bulk graphite are presented in Fig. 6. Maximums near the light wavelength $650 \mathrm{~nm}$ are visible on both curves (larger maximum values are observed for the graphene layer).

Graphene dispersion curves behavior near $650 \mathrm{~nm}$ (Figs 6 to 8 ) can be explained similarly to the results from $[11,12]$. The copper substrate is not smooth and contains granules on its surface with their own quantum confinement or plasmon oscillation frequencies. Electromagnetic waves with the frequency corresponding to the surface granules resonant frequencies induce electromagnetic oscillations, which result in total electromagnetic field intensity increase. The electromagnetic field increase develop an optical effect in molecules that are deposited on the metal substrate and are also close to the surface. In our experiments, we used graphene crystals on the copper surface. It may result in optical effects that can include Raman scattering, absorption or nonlinear optical effects. A higher level of absorption in the graphene layer is presented in Figs 6 to 8. In accord with $[11,12]$, the amplification coefficient $G$ can be introduced as:

$G=E / E_{0}$,

where $E$ is the total electric field in the vicinity of granules on the surface, $E_{0}$ - amplitude of the incident electromagnetic wave. Some of the reflected $p$ - and $s$ light wave components include plasmon osculations in the substrate surface region, which can result in high reflection coefficients $R_{p}$ and $R_{s}$. The latter results in increasing values of the absorption coefficient $k$ and optical conductivity $\sigma(\omega)$, too. The rise of the optical constants can be explained in frames of light propagation in metals basic theory. Accordingly to the work [11], where granules were taken as spheres, the amplification coefficient $G$ depends on the sphere diameter and has the maximum value at the $10-\mathrm{nm}$ diameter for $\mathrm{Ag}$ and $\mathrm{Au}$. We calculated maximum values of the amplification coefficient in our case when copper nanogranules are surrounded by graphene molecules. In this theoretical modeling, the nanogranules were taken as spheres having a certain diameter. STM experiments show the average diameter of the copper granules close to $0.3 \mathrm{~nm}$. The experiments show also that the ratio $q$ of volume filling by copper granules is approximately 0.7 . Due to [11], the maximal amplification coefficient can be found using the expression:

$G_{\max }=3\left|\varepsilon^{\prime}(\omega)\right| / \varepsilon^{\prime \prime}(\omega)$,

where $\varepsilon^{\prime}$ is a real part of complex dielectric permittivity, and $\varepsilon^{\prime \prime}$ is an imaginary part at $\omega$ light frequency. Because the calculated $q$ exceeds 0.5 in order to find dielectric permittivity components we applied Bruggemann expression:

$\left(\varepsilon_{2}-\varepsilon_{3}\right) /\left(\varepsilon_{2}+2 \varepsilon_{3}\right)=(1-q)\left(\varepsilon_{0}-\varepsilon_{3}\right) /\left(\varepsilon_{0}+2 \varepsilon_{3}\right)$, where $\varepsilon_{2}$ is the complex dielectric permittivity inherent to the heterogeneous layer of graphene and copper granules 2 in Fig. $1, \varepsilon_{0}$ and $\varepsilon_{3}$ are dielectric permittivities for graphene and copper, respectively (Fig. 1). The heterogeneous layer 2 in Fig. 1 is granules of copper covered by thin graphene layer.

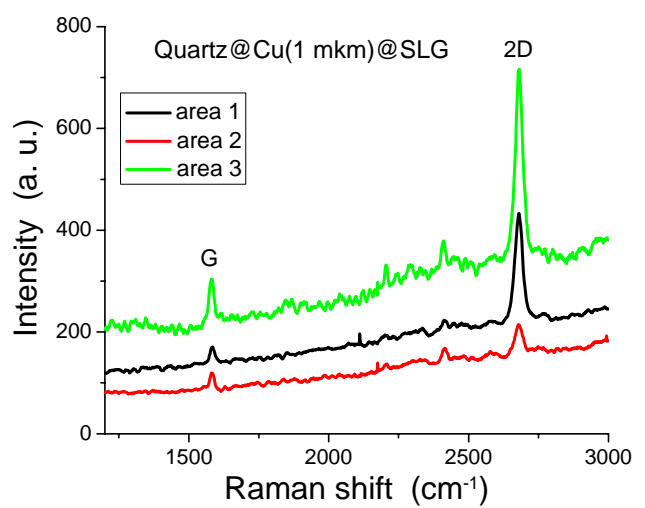

Fig. 5. Main Raman bands of graphene layer on top of $\mathrm{Cu}$ layer with the thickness $1 \mu \mathrm{m}\left(\lambda_{e x c}=514 \mathrm{~nm}\right)$.

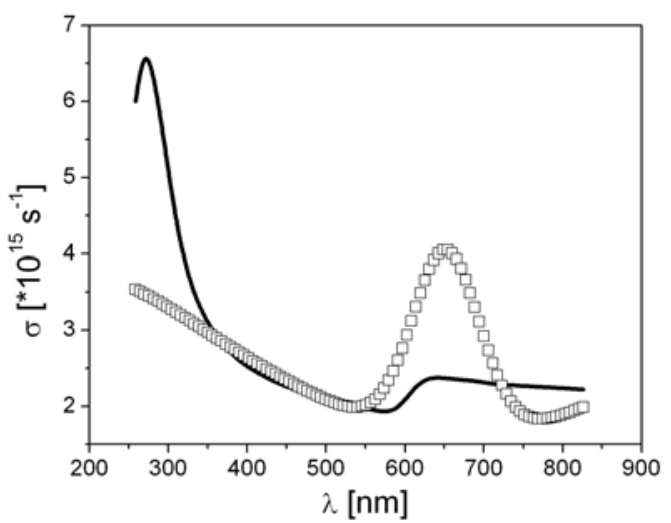

Fig. 6. Dispersion curves of calculated optical conductivity $\sigma$ for graphene on a copper substrate $(\square)$ and experimental data for bulk graphite (一).

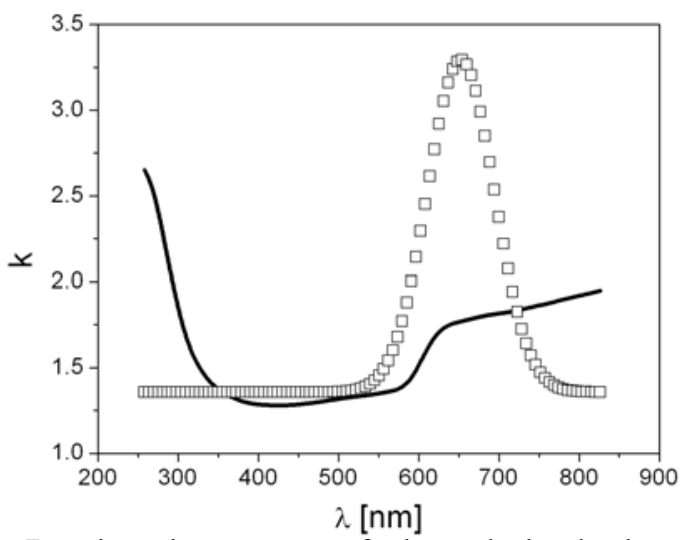

Fig. 7. Dispersion curves of the calculated absorption coefficient $k$ for graphene on the copper substrate ( $\square$ ) and experimental data for bulk graphite (一). 


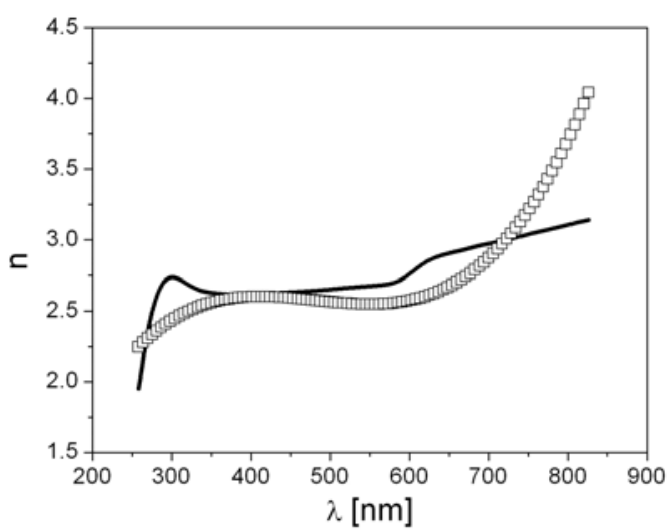

Fig. 8. Dispersion curves of the calculated refraction index $n$ for graphene on the copper substrate ( $\square$ ) and experimental data for bulk graphite (一).

We performed the calculations for the light wavelength $\lambda=652 \mathrm{~nm}$, because maximal values of optical conductivity and absorption coefficient are observed in this spectral region (Figs 6 and 7). Values for the layer 2 from Fig. 1, we took from [10], and layer 3 values were taken from ellipsometric measurements. The values for the ratio in (7) were taken as follows: $q=0.7$, $\varepsilon_{0}{ }^{\prime}=5.3, \varepsilon_{0}{ }^{\prime \prime}=10.3, \varepsilon_{3}{ }^{\prime}=-9.5, \varepsilon_{3}{ }^{\prime \prime}=0.49$. Applying the equations (6) and (7), we determined the maximal amplification coefficient $G_{\max }$ that was found to be equal to 3.1. Of course, we should realize the granules on the copper surface are not perfect spheres, and the upper estimate has to be taken as the first approximation. In order to find how electric field rise influences both optical conductivity and absorption of our sample, let us take the classic expression for the optical conductivity as a function of the light frequency [13]:

$$
\sigma(\omega)=N_{0} e^{2} f_{i} \omega^{2} \Gamma / m\left[\left(\omega_{0}{ }^{2}-\omega^{2}\right)^{2}+\omega^{2} \Gamma^{2}\right] .
$$

Here, $N_{0}$ is the number of oscillators per volume unit, $e$ - electron charge, $m$ - electron mass, $\omega_{0}-$ resonant frequency, $\Gamma$ - damping coefficient, and $f_{i}-$ oscillator strength which is defined as

$$
f_{i}=N_{i} / N_{0},
$$

here $N_{i}$ is the number of oscillators that absorb light, and $N_{0}$ - total number of oscillators.

Analyzing Eq. (8), we can conclude that the electric field value rise results in the drop of irradiation damping coefficient $\Gamma$. Lower values of $\Gamma$ lead to higher values of optical conductivity $\sigma(\omega)$. As a result, the rise of the electric field value results in longer lifetimes of electrons on the excited level, because $\Gamma$ - value is inversely proportional to the excitation level lifetime.

\section{Conclusions}

1. The dispersion curve of refraction index for thin graphene film on a copper substrate is similar to the bulk graphite spectral characteristics with almost identical features.

2. Absorption coefficient of the thin graphene film as well as optical conductivity dispersion curves has a strong absorption band. The band is observed near $650 \mathrm{~nm}$ in the spectral range that corresponds to a low maximum for bulk graphite in the same region. We observe significant enhancement of the absorption band from a graphene layer generated by near fields of plasmonic oscillations in $\mathrm{Cu}$ nanostructures. This enhancement has been confirmed by theoretical modeling.

\section{References}

1. X. Li, W. Cai, J. An et al., Large-area synthesis of high-quality and uniform graphene films on copper foils // Science, 324, p. 1312-1314 (2009).

2. S. Mao, K. Yu, J. Chang, D. Steeber, L. Ocola and J. Chen, Direct growth of vertically-oriented graphene for field-effect transistor biosensor // Sci. Repts. 3(1696), p. 1-6 (2012).

3. V.G. Kravets, R. Jalil, Y.-J. Kim et al., Grapheneprotected copper and silver plasmonics // Sci. Repts. 4 (5517), p. 1-8 (2014).

4. A. Hill, S.A. Mikhailov and K. Ziegler, Dielectric function and plasmons in graphene / A Letter Journal Exploring the Frontiers of Physics // Europhys. Lett. 87, 27005 (2009).

5. C. Tegenkamp, H. Pfnuer, T. Langer, J. Baringhaus and H. Schumacher, Plasmon electron-hole resonance in epitaxial grapheme // J. Phys.: Condens. Matter, 23, 012001 (2011).

6. S. Bae, H. Kim, Y. Lee et al., Roll-to-roll production of 30-inch graphene films for transparent electrodes // Nat. Nanotech. 5, p. 574 (2010).

7. J.R. Beattie, Optical constants of metals in the J.R. Experimental methods // Phil. Mag. 46, p. 235-245 (1955).

8. M. Born, E. Wolf, Principles of Optics. Cambridge, 2002.

9. A.C. Ferrari, J.C. Meyer, V. Scardaci et al., Raman spectrum of graphene and graphene layers // Phys. Rev. Lett. 97, 187401 (2006).

10. E.D. Palik, Handbook of Optical Constants of Solid. Vol. 1. Academic Press London, Boston, New York, 1998.

11. E.F. Venger, A.V. Goncharenko, M.L. Dmitruk, Optics of Small Particles and Disperse Media. Kyiv, Naukova Dumka, 1999 (in Russian).

12. V.A. Kosobukin, Collective effects in amplification of external electrical field on surface of metals // Izvestia AN SSSR, ser. fiz. 9, №6, p. 1111-1112 (1985), in Russian.

13. A.V. Sokolov, Optical Properties of Metals. American Elsevier Pub. Co., 1967. 